\title{
Wearable device for remote monitoring of transcutaneous tissue oxygenation
}

\author{
Juan Pedro Cascales, (D) Emmanuel Roussakis, Lilian \\ Witthauer, (D) Avery Goss, Xiaolei li, Yenyu Chen, Haley L. \\ MARKS, (D) AND CONOR L. EVANS*
}

Wellman Center for Photomedicine, Massachusetts General Hospital, Harvard Medical School, Charlestown, Massachusetts 02129, USA

*evans.conor@mgh.harvard.edu

\begin{abstract}
Wearable devices have found widespread applications in recent years as both medical devices as well as consumer electronics for sports and health tracking. A metric of health that is often overlooked in currently available technology is the direct measurement of molecular oxygen in living tissue, a key component in cellular energy production. Here, we report on the development of a wireless wearable prototype for transcutaneous oxygenation monitoring based on quantifying the oxygen-dependent phosphorescence of a metalloporphyrin embedded within a highly breathable oxygen sensing film. The device is completely self-contained, weighs under 30 grams, performs on-board signal analysis, and can communicate with computers or smartphones. The wearable measures tissue oxygenation at the skin surface by detecting the lifetime and intensity of phosphorescence, which undergoes quenching in the presence of oxygen. As well as being insensitive to motion artifacts, it offers robust and reliable measurements even in variable atmospheric conditions related to temperature and humidity. Preliminary in vivo testing in a porcine ischemia model shows that the wearable is highly sensitive to changes in tissue oxygenation in the physiological range upon inducing a decrease in limb perfusion.
\end{abstract}

(C) 2020 Optical Society of America under the terms of the OSA Open Access Publishing Agreement

\section{Introduction}

Wearable devices that provide continuous monitoring of physiological variables have found widespread application in recent years as both medical devices and in consumer electronics for sports and health tracking. Commercially available devices can measure a wealth of variables such as heart or respiration rate, blood oxygen saturation, motion, force, temperature, muscle activation, etc. [1,2]. However, commercial wearables still lack essential capabilities to measure several important physiological parameters.

Oxygen concentration is a crucially important parameter that often goes unmeasured, despite the fact that it constitutes a key component in the cellular energy production machinery. Knowledge of tissue oxygen tension or oxygen partial pressure $\left(\mathrm{pO}_{2}\right)$ at the skin surface can be decisive for diagnostic applications in burns, limb injury and surgical interventions [3-6]. To this end, blood oxygen saturation measurement $\left(\mathrm{stO}_{2}\right)$ methods are often used [7]. However, these saturation measurements are indirect measures of tissue oxygen content and do not provide accurate readings when blood flow is impaired [8,9]. In contrast, transcutaneous oxygen tension measurements (TCOM) are direct measurements of the oxygen available in the tissue ready to be metabolized by cells and do not solely depend on oxygen delivery by blood or the condition of the underlying tissue capillary bed. TCOM devices measure local oxygenation of the tissue and can be advantageous in situations such as the application of tourniquets [10] or hyperbaric treatments [11], as measurement of $\mathrm{pO}_{2}$ could indicate the onset of tissue ischemia or oxygen poisoning, respectively. Blood oxygen saturation, on the other hand, would lose the ability to measure further changes in these scenarios upon reaching values of $0 \%$ or $100 \%$ in each case. 
Despite its advantages compared to devices that measure oxygen saturation, measurements of $p \mathrm{O}_{2}$ and oxygen tension are not widely utilized mainly because traditional TCOM technology involves the use of bulky equipment, time-consuming and frequent bedside calibration, precise placement, and well-trained operators. An ideal approach to achieve truly portable technology for tissue oxygenation monitoring is to create optical tools based on metalloporphyrin molecules which undergo phosphorescence quenching by oxygen $[12,13]$. Previous efforts exploiting this principle were successful in creating sensors for transcutaneous oxygen measurements which were responsive to changes in the lifetime of oxygen-sensing phosphors incorporated within transparent films $[14,15]$. Nonetheless, the ability to detect phosphorescence lifetime often comes at a price as it typically requires cumbersome experimental techniques and large benchtop instrumentation [16,17]. Early attempts to improve the portability, ease-of-use, and data gathering capabilities of TCOM technologies involved the use of oxygen sensing molecules in studies in which 2D maps of the local oxygenation and oxygen consumption rate of tissue were generated by collecting luminescence intensity images with a modified digital camera [3,18-22]. Intensity-based approaches can show excellent performance in specific scenarios with controlled experimental conditions or settings [3,20,23]. Work carried out by our group with a paint-on oxygen sensing bandage [22] found that the transdermal monitoring of oxygenation was able to detect limb ischemia when arterial ligation was performed on a rat model, proving that transdermal oxygenation can be a non-invasive alternative to measuring tissue health. Techniques sensitive to phosphorescence lifetime, on the other hand, exhibit significant advantages as they typically do not experience signal variability from changes in sensor geometry, excitation source intensity, photobleaching, etc. that can plague intensity-based methods. Current advances in low-power electronics, communications and materials engineering have contributed to the development of TCOM wearables, however, these currently available devices actually only consist of wearable-like sensing heads that ultimately rely on large external readout electronics [24-30] or are standalone devices but involve invasive components or procedures [31-34].

In this manuscript, we report on the development of a wireless and non-invasive wearable prototype that aims to continuously monitor tissue oxygenation based on in-house developed metalloporphyrins [35], which can be readily embedded within polymer films, thus providing materials that exhibit bright emission throughout the physiological $\mathrm{pO}_{2}$ range.

\section{Results}

\subsection{Response and calibration}

The wearable device measures tissue oxygenation at the skin surface by detecting changes in lifetime $\tau$ and intensity $I$ of the phosphorescence emission of a highly breathable multilayer oxygen sensing film. The film exhibits bright luminescence in the $\mathrm{pO}_{2}$ range $0-160 \mathrm{mmHg}$ with a peak emission at $650 \mathrm{~nm}$. It yields a lifetime of approximately $15 \mu \mathrm{s}$ in room air $\left(\mathrm{pO}_{2}=160 \mathrm{mmHg}\right)$ and approximately $96 \mu$ s at zero oxygen (see Fig. S1). The oxygen-sensing film is composed of several layers (see Fig. 1 for film orientation): a semi-permeable transparent membrane which partially isolates the skin from atmospheric oxygen (similar to approaches found in Refs [25-27]), a poly(propyl methacrylate) (PPMA) [36] film with embedded metalloporphyrins, a transparent and breathable membrane, and a spin coated, white breathable layer which increases the collected emission through back-scattering while also serving as optical insulation from external light sources. The films are impervious to changes in relative humidity [37], solving one of the key challenges faced when implementing these oxygen sensing materials into wearable devices monitoring human performance, where sweat can vary widely depending on climatological conditions or body location placement [38].

As shown in Fig. 1, the device is built around a wireless enabled microcontroller and consists of a small sensor head and the main control electronics. The adhesive oxygen sensing film is affixed onto the sensor head, which is $14 \mathrm{~mm}$ in diameter and $3 \mathrm{~mm}$ in thickness and is comprised of a 


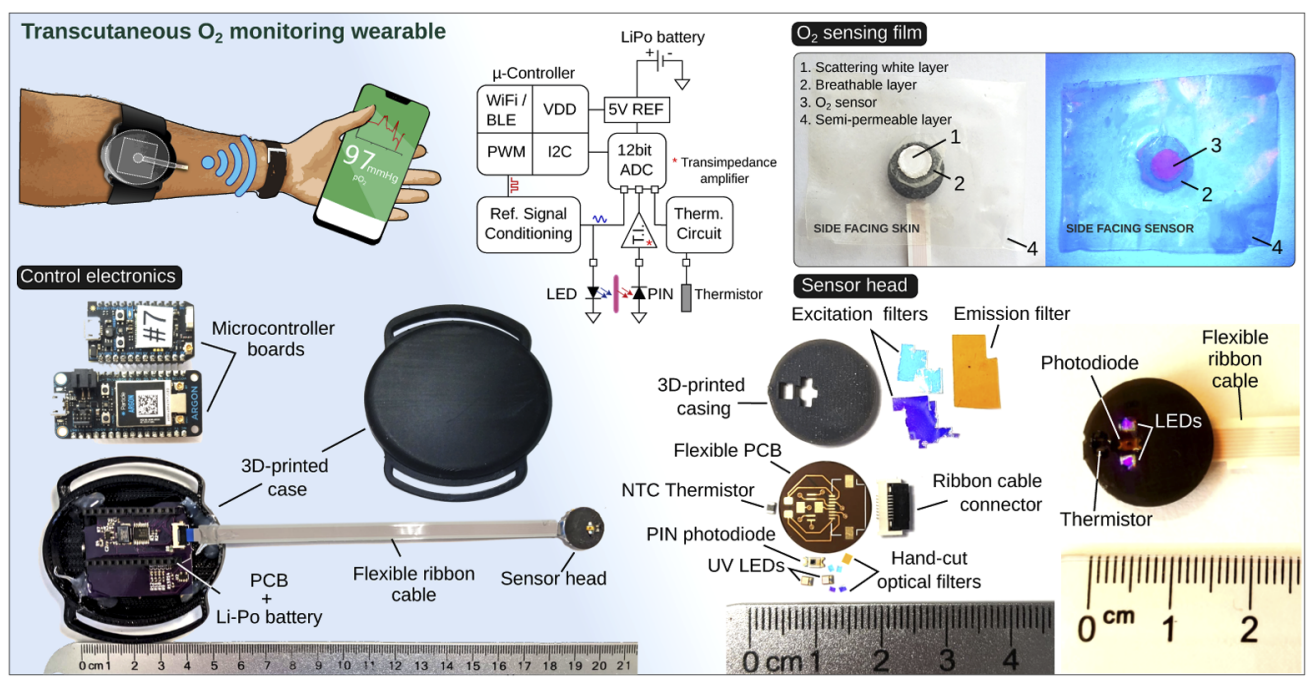

Fig. 1. Optical wireless wearable prototype for transcutaneous oxygen monitoring based on the phosphorescence emission of a highly breathable oxygen sensing film. The prototype is composed of an oxygen-sensing film, a sensor head, and control electronics. The block diagram represents the control electronics and sensor head circuits.

flexible printed circuit board (PCB) within a 3D printed casing. The PCB is host to several small surface-mount electronics: two high power UVA LEDs (Lumileds, Amsterdam, Netherlands) with a peak wavelength at $385 \mathrm{~nm}$ to excite the phosphor molecules and generate emission from the oxygen-sensing film, a PIN photodiode (Osram, Munich, Germany) to detect the emission, and a thermistor (TDK, Tokyo, Japan) to measure temperature. The LED excitation is filtered by stacking two ultra-thin flexible optical notch-filters (Edmund Optics, Barrington, NJ, USA), which serve as a $400 \mathrm{~nm}$ short-pass filter in the range of interest (see Fig. S2). A $500 \mathrm{~nm}$ long-pass collection filter, which blocks the LED emission, is fabricated by combining a flexible $405 \mathrm{~nm}$ long-pass filter (Edmund Optics, Barrington, NJ, USA) and a polyamide film (DuPont, Wilmington, DE, USA) (see Fig. S2). The sensor head is attached to the control electronics via a thin, flexible ribbon cable. By folding the flexible connector and the sensor head under the control electronics case, the sensor head and oxygen sensing film are shielded from mechanical stress. This design helps to both protect the sensor head and provide adhesion, as well as an airtight and stable seal of the $\mathrm{pO}_{2}$ sensing film over the skin (see Fig. 1). The device is a true wearable in that it is entirely self-contained, performs the signal analysis on-board and weighs only about $30 \mathrm{~g}$.

As shown in the block diagram in Fig. 1 and the ADC output waveforms shown in Fig. 2(b), the device detects changes in phosphorescence lifetime of the oxygen sensing phosphor by driving the excitation LEDs with a sinusoidal-like reference signal $\left(f_{r}=796 \mathrm{~Hz}\right)$ and measuring the microsecond delay or phase $(\theta)$ between the reference signal and the emission signal, also a sinusoidal $[16,17,24,39-43]$. In the case of only a single lifetime being present in the emission, the phase can be related to lifetime by the expression $\tan (\theta)=2 \pi \tau f_{r}$ [16]. Because our film contains a single phosphorescent dye in which $97 \%$ of the molecules exhibit the same lifetime (see Fig. S1), a sinusoidal reference signal is sufficient to fully characterize the phosphorescence lifetime. As described below, this allows for a simple signal analysis approach which can be performed on-board a device with limited computing power such as that of microcontrollers. The change in lifetime $\tau$ is also reflected by the intensity $I$ of the emission, which responds to variations in $\mathrm{pO}_{2}$, and is obtained from the amplitude of the measured signal at the driving 


\section{Biomedical Optics EXPRESS}

frequency. An algorithm based on Multiple Linear Regression in the matrix form [44] was developed to extract lifetime and intensity of the emission (see Methods).
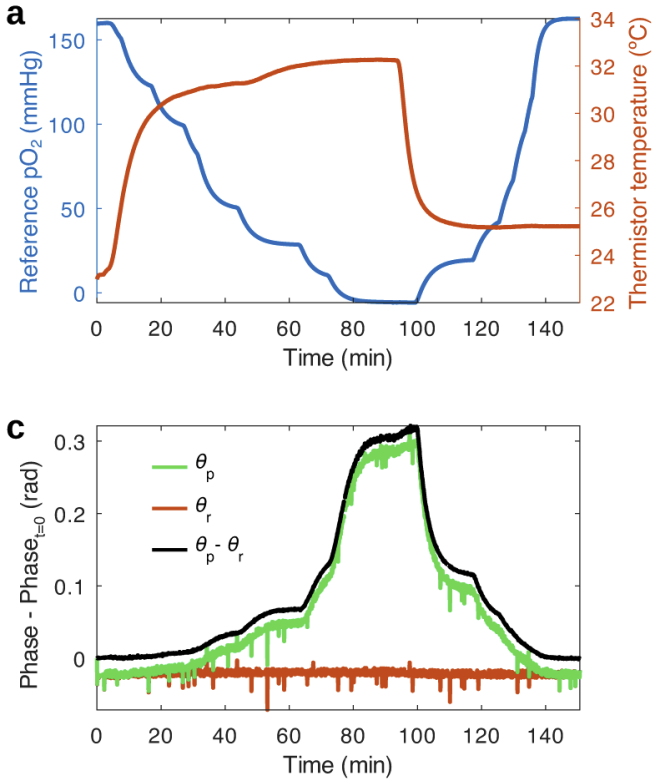
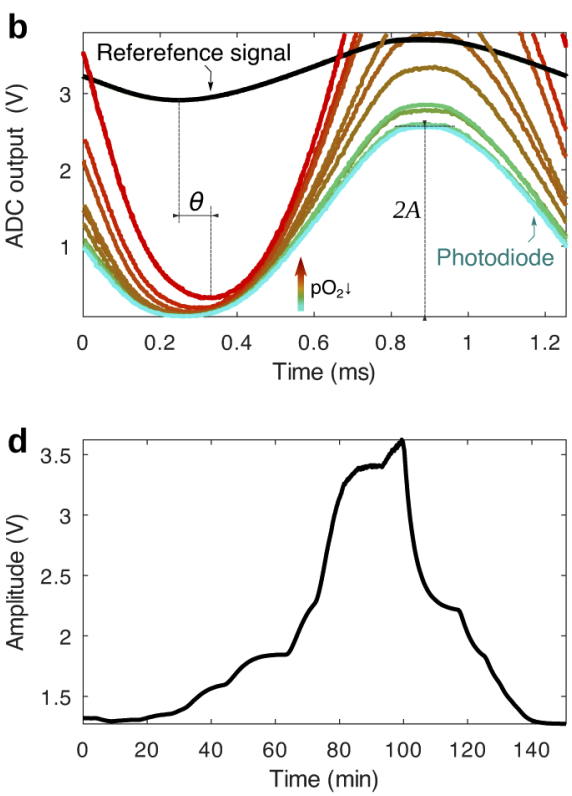

Fig. 2. Response of the oxygen sensing film to $\mathrm{pO}_{2}$ and temperature during a calibration run. (a) Variation of the temperature (measured by the thermistor in the sensor head) and $\mathrm{pO}_{2}$ (measured by a commercial oxygen sensor) in a sealed chamber during calibration, in which the oxygen partial pressure is varied by mixing nitrogen and air at different ratios. (b) ADC output of the photodiode and reference signal channels at different points in time throughout the calibration. The photodiode signal reveals how the phosphorescence of the oxygen sensing film changes in amplitude and phase with respect to the reference signal during changes in oxygen (and temperature to a lesser degree). The reference signal remains stable during the measurement. (c) Phase (minus the initial value at $t=0$ ) of the reference and photodiode signal vs time during the calibration period. The relative phase between the photodiode and reference signal, $\theta=\theta_{p}-\theta_{r}$, exhibits high sensitivity to changes in $\mathrm{pO}_{2}$ throughout the whole physiological range. (d) Amplitude of the emission $I$ vs time, presenting a similar response to the phase. Phase and amplitude are obtained with a multiple linear regression algorithm from the data in (b).

The dependence of the measured variable $X$, namely $\tau$ or $I$ with $p O_{2}$, is governed by this equation, modified from the Stern-Volmer relation [45]:

$$
X=X_{0} /\left(1+K_{e f f} \cdot p O_{2}\right)+X_{O F F}
$$

where $X_{0}$ is the value of $X$ in the absence of oxygen and $X_{O F F}$ is a non-oxygen dependent offset arising from the measurement system. In the case of dynamic quenching and diffusioncontrolled processes, the Stern-Volmer quenching constant, $K_{\text {eff }}$ which is a measure of the oxygen diffusion coefficient of the multilayer film. $K_{\text {eff }}$ is temperature dependent mainly because of the collisional quenching rate of the porphyrin [38] and to a lesser degree, due to changes in the oxygen-diffusion parameters of the polymer layers [46], which may also experience changes through aging as was found below. A quadratic dependence of $K_{\text {eff }}$ with temperature $\left(K_{\text {eff }}=K_{0}+K_{1} \cdot(T-32)+K_{2} \cdot(T-32)^{2}\right)$ was found, which accounts for the trends observed in the data measured with prototypes fabricated with the final design seen in Fig. 1. Earlier iterations of the device in which different 3D printed materials were used yielded a negligible quadratic 
term, which can be seen in the data shown in Table 1, and is explained by slight differences in sensor geometry, LED leakage into the photodiode signal and thermal properties of the sensor head.

\begin{tabular}{|c|c|c|c|}
\hline Parameter & Initial & Re-positioned & Aged (3 months) \\
\hline$\theta_{0}$ & 0.309 & $0.302(-2.1 \%)$ & $0.317(+4.8 \%)$ \\
\hline$\theta_{\text {OFF }}$ & 1.005 & $0.999(-0.6 \%)$ & $1.002(+0.3 \%)$ \\
\hline$K_{0}$ & 0.0108 & $0.0114(+5.6 \%)$ & $0.0086(-24.6 \%)$ \\
\hline$K_{1}$ & 0.00056 & $0.00058(+3.6 \%)$ & $0.00041(-29.3 \%)$ \\
\hline$I_{0}$ & 1.043 & $0.848(-18.7 \%)$ & $1.323(+56.0 \%)$ \\
\hline$I_{O F F}$ & 0.292 & $0.289(-1.0 \%)$ & $0.328(+13.5 \%)$ \\
\hline$K_{0}$ & 0.0822 & $0.0756(-8.0 \%)$ & $0.0821(+8.6 \%)$ \\
\hline$K_{1}$ & 0.00236 & $0.00214(-9.3 \%)$ & $0.00206(-3.7 \%)$ \\
\hline
\end{tabular}

To evaluate the performance of the prototype, the sensor head was outfitted with an oxygensensing film and the device was inserted into a sealed calibration chamber (see Fig. S3). The variation of temperature and oxygen partial pressure during calibration is shown in Fig. 2(a), during which a first sweep of $p \mathrm{O}_{2}$ is carried out at a high body-like temperature and a second one is performed at room temperature. As can be seen in in Fig. 2(b) and (c), $\theta_{p}$ monotonically increased as $\mathrm{pO}_{2}$ was gradually swept between atmospheric values $(160 \mathrm{mmHg})$ and a pure nitrogen atmosphere $(0 \mathrm{mmHg})$. Throughout the measurement, temperature was varied between room conditions $\left(\sim 24^{\circ} \mathrm{C}\right)$ and the temperature of skin $\left(\sim 32{ }^{\circ} \mathrm{C}\right)$. Both $\theta_{p}$ and $\theta_{r}$ present similar fluctuations, which stem from slight differences between $\mathrm{pO}_{2}$ measurements in the time elapsed from the PWM output turning "ON" to the ADC sampling start. The fluctuations in $\theta_{p}$ can be removed by calculating the relative phase change between photodiode and reference signals, i.e. $\theta=\theta_{p}-\theta_{r}$, referred to as the phase from here on. The intensity of the emission, as seen in Fig. 2(d), shows a very similar response. Both the lifetime and intensity signals present high signal to noise ratio ( $29 \mathrm{~dB}$ and $31 \mathrm{~dB}$ respectively) and require no smoothing or filtering in order to achieve a good signal contrast throughout the physiological range. These results were consistent throughout multiple prototypes and $\mathrm{O}_{2}$-sensing films.

Fig. 3(a),(b) and (d),(e) show that features in lifetime and intensity data due to changes in $\mathrm{pO}_{2}$ and temperature, respectively, are well described by the temperature dependent Stern-Volmer equation. The estimate of $\mathrm{pO}_{2}$ obtained from lifetime and intensity data, shown in Fig. 3(c) and (f), closely matches what is measured with a reference commercial $p O_{2}$ sensor, with discrepancies possibly arising from a difference in response time of both devices and from each sensor experiencing different temperature gradients due to their location within the chamber.

In order to test for reproducibility of the calibration parameters related to the positioning of the sensing film, an already calibrated oxygen sensing film was removed from the device and re-affixed onto the sensor head. The subsequent calibration showed that all the fitting parameters (see Table 1) obtained for lifetime differed very little from the previous values. However, the fitting parameters for intensity were observed to vary with respect to the earlier position of the oxygen sensing film. The invariance of lifetime parameters against sensor placement highlights a 


\section{Biomedical Optics EXPRESS}
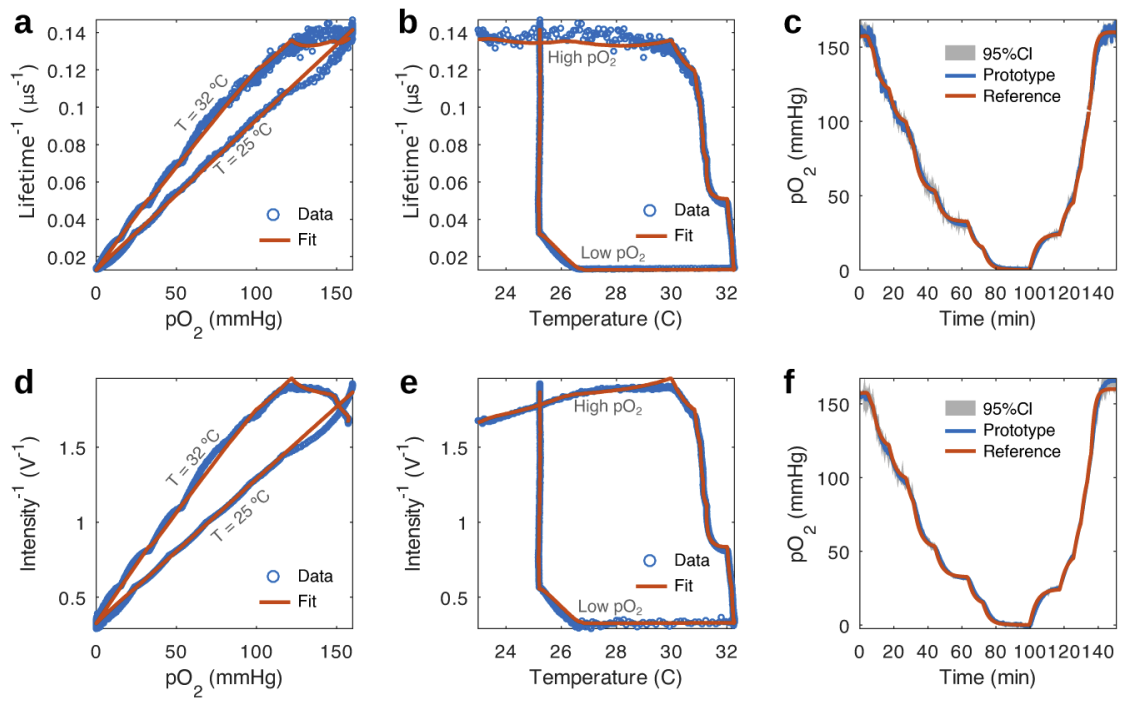

Fig. 3. Fits of the temperature dependent Stern-Volmer equation. (a) Stern-Volmer plot of lifetime data and its fit to the temperature dependent Stern-Volmer equation. The model is able to describe the variation of the measured lifetime with changes in partial pressure of oxygen and temperature. (b) Complimentary lifetime data vs temperature. The dependence of lifetime with temperature is accounted for by modelling $K_{\text {eff }}$ as a second order polynomial depending on temperature. (c) Comparison of $\mathrm{pO}_{2}$ measured by the developed device and a commercial reference sensor along with a $95 \%$ confidence interval (CI) of measurements. The $\mathrm{pO}_{2}$ estimated from lifetime data reproduces all features observed in the reference $\mathrm{pO}_{2}$ data with slight differences attributed to mismatches in sensor speed and temperature compensation. (d), (e) and (f) show equivalent plots for the intensity data revealing similar features.

key advantage of lifetime-based measurements over intensity-based approaches, which are highly dependent on orientation.

We also tested the aging of the film with time (see Table 1) by storing the device with a film attached for three months, such that it was kept shielded from room lighting but not from changes in ambient conditions. A calibration of the film revealed that $\theta_{0}$ and $\theta_{O F F}$ parameters remained unchanged with respect to the previous calibration, while $K_{0}$ and $K_{1}$, experienced a large decrease, which may point to the film's oxygen diffusion properties being modified with passing time. The intensity parameters did not exhibit a clear trend.

\subsection{Sensing tissue oxygenation in an in vivo porcine model}

To evaluate the response of the device in monitoring tissue oxygenation during physiological changes, an in vivo experiment was conducted on a porcine model (Yorkshire swine). Porcine anatomy and specifically their skin is highly similar to that of humans (structure, thickness, etc.) which makes the porcine model suitable to monitor physiological oxygenation changes transdermally. Changes in tissue oxygenation were induced by occluding blood flow to the front limb of the porcine model by applying a tourniquet above the elbow joint (over the triceps, brachii, and brachialis muscles), as shown in Fig. 4(a), for a duration of 30 minutes. The 30 minute waiting period was chosen guided by previous work [22], to allow for the device to equilibrate to skin $\mathrm{pO}_{2}$. The animal surgery protocol was reviewed and approved by the Institutional Animal Care and Use Committee (IACUC) at Massachusetts General Hospital. 


\section{Biomedical Optics EXPRESS}

a

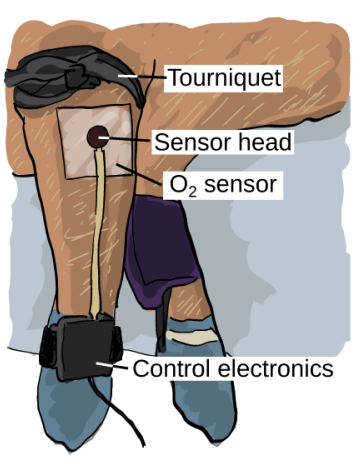

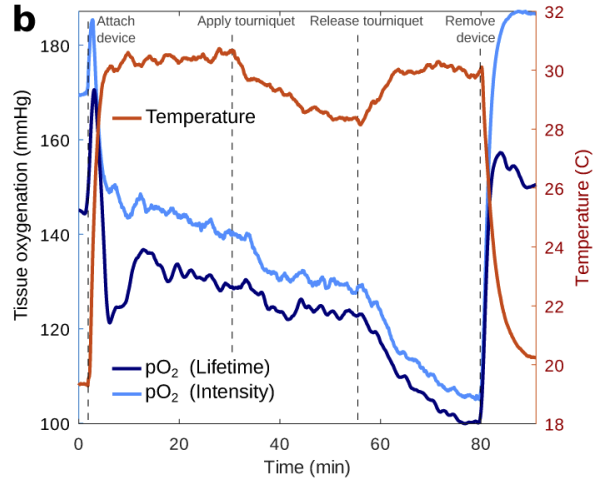

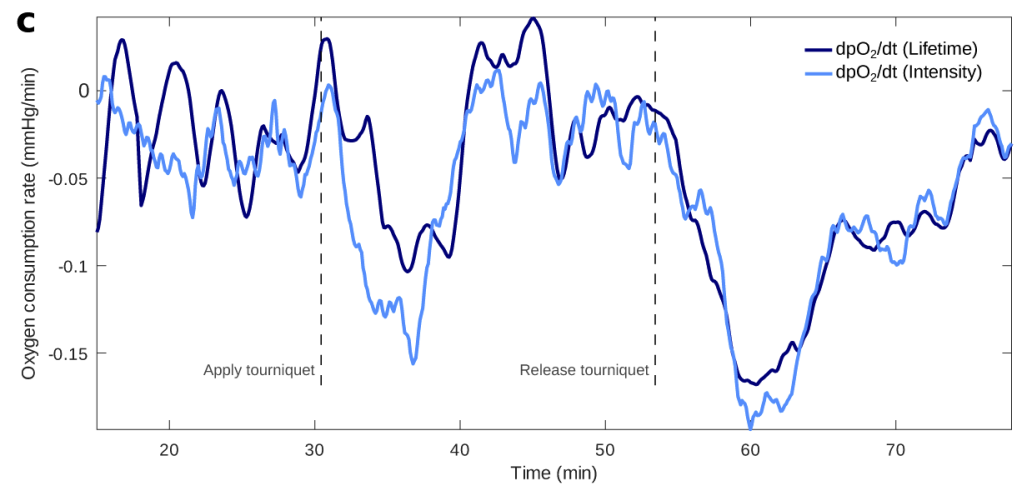

Fig. 4. In vivo testing of the oxygen sensing prototype in a porcine model. (a) Photograph of the experimental set-up. Blood flow was occluded in a front limb of a Yorkshire swine by applying a tourniquet over the elbow joint to induce changes in tissue oxygenation. The wearable was placed over a shaved area of skin on the upper limb. (b) Temperature and $\mathrm{pO}_{2}$ (estimated from lifetime and intensity) during the experiment. As seen in both the estimates of $\mathrm{pO}_{2}$ and temperature, the device is sensitive to physiological changes due to the compromised blood flow to the limb during the full occlusion. (c) Time derivatives of both partial oxygen pressure estimates reveal a faster rate of change of the local oxygenation for the minutes following application and removal of the tourniquet.

To prepare the region of interest, a 10x10 cm area of the swine's front upper extremity was cleaned with soapy water and shaved until all hair was removed. After shaving, the skin was wiped with isopropranol. The oxygen sensing film was pre-fixed onto the sensor head and atmospheric $\mathrm{pO}_{2}$ was sampled for several minutes as a reference prior to applying the device onto the skin, as seen in Fig. 4(b). The reference reading differed from $160 \mathrm{mmHg}$ due to the starting temperature $\left(19{ }^{\circ} \mathrm{C}\right)$ being outside the range of temperatures considered during calibration $\left(23-32{ }^{\circ} \mathrm{C}\right)$. As seen in the data, lifetime $\mathrm{pO}_{2}$ showed room air values close to $160 \mathrm{mmHg}$ when temperature was within the calibration range $\left(T \geq 23^{\circ} \mathrm{C}\right)$. This issue is easily solved by expanding the calibrated temperature range. Upon application of the device onto the pig's skin, the sensor head quickly achieved thermal equilibrium in approximately 3 min due to its small mass. Both estimates of $\mathrm{pO}_{2}$ as obtained from phosphorescence lifetime and intensity, exhibited an exponentially decaying trend due to a number of factors such as initial oxygen trapped between film and tissue, tissue oxygenation, which is a balance between oxygen supplied by the blood and oxygen consumed by the muscle and skin, atmospheric oxygen uptake by the skin, skin oxygen permeability, and atmospheric oxygen permeability through the medical adhesive layer. 
After a 30 minute equilibration period, the tourniquet was applied and both lifetime and intensity $\mathrm{pO}_{2}$ metrics were observed to respond immediately to the compromised blood flow by decreasing at a faster rate (Fig. 4(b)). This is clearly seen by plotting the time derivative of $\mathrm{pO}_{2}$, shown in Fig. 4(c), which is indicative of oxygen consumption rate in a tourniquet scenario. The data revealed an increasingly negative $\mathrm{pO}_{2}$ slope that reached its maximum value at around 5 minutes into the application of the tourniquet (occlusion). Due to the lack of blood flow, the temperature of the limb decreased by as much as $2.5^{\circ} \mathrm{C}$ from the original baseline temperature. The tourniquet was released after 30 minutes and the temperature of the limb was observed to return to baseline while $\mathrm{pO}_{2}$ once again exhibited an increased rate of decay for around 5 minutes. This further decay, which occurred alongside intense blanching of the limb's skin, could indicate the presence of irreversible tissue damage due to the tourniquet's placement.

\section{Discussion}

These preliminary results demonstrate the device's great potential for applications involving monitoring oxygenation in living tissue. Despite their enhanced capabilities as diagnostic or monitoring tools for predicting wound healing, determining amputation level, hyperbaric oxygen therapy, severity of ischemia, etc., devices which directly measure tissue oxygenation have not found widespread use due to issues such as the need for unwieldy tools, complex calibration procedures, and extensive user training. The developed wearable device overcomes these issues by using readily available, low-power electronic components that can be easily interfaced with multiple devices such as computers or smartphones. The device requires very few components and its size could be greatly reduced for commercial application. As such, the technology described here could certainly find application in consumer devices, therefore allowing the assessment of tissue oxygen at the point of care, as well as being accessible to those in low resource settings. Furthermore, the algorithm we developed to extract lifetime and intensity from the time series is ideally suited for implementation in embedded devices with low computing power as it involves simple matrix operations with minimal memory requirements.

The measurement is consistently reproducible between devices and calibrations, and is robust against changes in temperature and humidity [37]. Because measurements of $\mathrm{pO}_{2}$ are based on lifetime detection, the device presents an important advantage over purely intensity-based measurements that may exhibit instabilities due to factors such as background noise, excitation source fluctuations and photobleaching. These claims are supported by the lifetime calibration parameters remaining constant regardless of film repositioning, which makes the lifetime based measurement more tolerant to motion. Additionally, as lifetime is not affected by parameters including film placement and LED intensity, the developed device can detect and compensate for effects related to the aging of the polymer film by tracking changes in permeability through the $K_{\text {eff }}$ constant. On the other hand, as seen in Table 1, such information could not be obtained from the intensity calibration parameters, as the overall response of intensity with oxygen changed with film aging, which affected film dryness and aggregation, amongst other elements. Furthermore, the lifetime estimate from the developed inexpensive and miniature device agrees well with the lifetime characterization carried out on the oxygen-sensing film with a state-of-the-art spectrometer (FLS1000 Steady State and Phosphorescence Lifetime Spectrometer, Edinburgh Instruments, Livingston, UK); the values for atmospheric and zero oxygen at $24{ }^{\circ} \mathrm{C}$ for the device are 10 and $80 \mu \mathrm{s}$, while those obtained with the spectrometer are 15 and $96 \mu$ s. The difference could be due to excitation light leaking into the detector [47] (see Fig. S9) or the presence of more than one lifetime species (see Fig. S1), which can result in the detection of a lower average lifetime [48]. These contributions could be taken into account with an appropriate model to better estimate the lifetime, however, we are able to obtain a reproducible relation between the phase and $\mathrm{pO}_{2}$ which was the main objective of the project. 
This study also proves the feasibility of the device's application in in vivo measurements on a porcine occlusion model. After inducing physiological changes, our device was capable of monitoring variations in tissue $\mathrm{pO}_{2}$ on the skin surface, responding quickly to changes in local oxygenation. Changes in variables such as temperature, humidity and skin blanching can confound $\mathrm{pO}_{2}$ measurements based on oxygen quenching of phosphorescence and are often overlooked or ignored in literature, or are overcome by adding heating elements [25-27] which prohibits the application of such tools, for example, in neonatal care. The device presented here was able to track changes in tissue oxygenation during an in vivo experiment without the measurement being affected by these variables. For a non-temperature compensated system, an increase in temperature could be misinterpreted as an increase in $\mathrm{pO}_{2}$. During in vivo testing, $p \mathrm{O}_{2}$ was observed to decrease faster upon both the application and removal of the tourniquet, which cannot be explained by the changes in limb temperature alone. We did not consider colder temperatures during the calibration of the film that was used for the in vivo study, but instead focused on warmer body-like temperatures. To address this in future studies, the sensor can be simply re-calibrated by sweeping a wider range of temperatures, which can be done using our calibration chamber, a hot plate and ice/water mixtures.

The device shows an ideal response time to detect physiological changes, which typically occur in the timescale of minutes. The fact that each component of the device was designed to be biocompatible (or skin-friendly) will accelerate the pace of translation as this wearable tool is brought into first-in-human clinical studies. Since the measurement is performed on the skin surface, with both skin and muscle contributing to the signal, and the little homogeneity which exists in how transcutaneous measurements are used to asses limb ischemia [49], the interpretation of transdermal oxygen measurements is complex and challenging, with many physiological factors which can contribute to the signal which are not yet well understood today. Future work will aim at designing clinical studies to disentangle the contributions of skin and muscle to the transcutaneous oxygenation signal, measuring tissue oxygenation during strenuous exercise, postoperative monitoring of transplanted tissue, and for the presence of bacterial infections. Further development of the device will involve miniaturization, targeting multiple dyes to monitor other physiological variables, implementing sensor fusion algorithms to combine the advantages of lifetime and intensity or apply machine learning to improve the prediction of $\mathrm{pO}_{2}$ values.

\section{Methods}

\subsection{Oxygen-sensing film}

\subsubsection{Chemicals and adhesives}

The pivaloyl-terminated, platinum porphyrin embedded within the oxygen-sensing film was prepared according to the protocol previously published by Roussakis et al [35]. Poly(propyl methacrylate) (PPMA) was purchased from Scientific Polymer Product. The adhesives used for the semi-permeable membrane ( $3 \mathrm{M}^{T M}$ Medical Tape 1513, Double Sided Transparent Polyester, 80\# Liner, Configurable) and the breathable membrane (3M ${ }^{T M}$ Medical Transfer Adhesive 1524, Fiber Filled Polyester, 60\# Liner, Configurable) were purchased from $3 \mathrm{M}$. The $\mathrm{TiO}_{2}$ white pigment concentrate and (45-55\% methylhydrosiloxane)-dimethylsiloxane copolymer (HMS) were purchased from Gelest. Platinum(0)-1,3-divinyl-1,1,3,3-tetramethyldisiloxane complex solution (platinum catalyst) was purchased from Sigma-Aldrich.

\subsubsection{Oxygen-sensing PPMA layer}

$0.05 \mathrm{mg} / \mu \mathrm{l}$ PPMA in dichloromethane was prepared in a small, clean Eppendorf tube. An aliquot from a stock solution of pivaloyl platinum porphyrin, such that the concentration of metalloporphyrin in the final solution was $30 \mu \mathrm{M}$, was added to the PPMA solution and mixed thoroughly by vortexing. $20 \mu \mathrm{l}$ of porphyrin/PPMA solution was deposited into an $8 \mathrm{~mm}$ diameter 
circular mold made with PDMS on a glass slide. The PPMA oxygen-sensing film was removed from the glass slide after drying in the hood for 30 minutes.

\subsubsection{White scattering layer}

$1 \mathrm{~g}$ of white pigment concentrate was mixed thoroughly with $3 \mu \mathrm{l}$ of platinum catalyst on a weighing boat. The mixture was added to a glass slide and spin coated for 1 minute at 1,500 rpm. $0.1 \mathrm{~g}$ of HMS copolymer was added on top of the white pigment film and spin coated for 1 minute at $750 \mathrm{rpm}$. The white scattering layer was allowed to fully cure overnight.

\subsection{Experimental design}

\subsubsection{Sensor head}

The flexible PCBs were designed with the KiCad software and fabricated with polyimide flexible substrates (OSH Park, Portland, Oregon, USA). The casing was fabricated with a Formlabs Form 3B 3D printer at resolution of $25 \mu \mathrm{m}$ utilizing a biocompatible photopolymer resin suited for medical applications. The fabricated parts were then washed in a 90-100\% isopropyl alcohol (IPA) bath for 20 minutes agitated by a magnetically coupled impeller and LED/heat cured at a wavelength of $405 \mathrm{~nm}$ for 30 minutes at $60^{\circ} \mathrm{C}$. Post curing, the fabricated parts could then be sterilized chemically or by autoclave. This component served as an optical mask for mounting the excitation and emission filters. It also served to optically isolate the LEDs from the photodiode, and as a skin-friendly, flat surface over which to place the oxygen-sensing film. The optimal geometry was found to be a planar configuration of the LEDs and photodiode, and to have the components as close as possible. The casing was attached to the PCB with UV-curing epoxy (Thorlabs, Newton, NJ, USA), which provided mechanical stability and optical transparency for the propagation of excitation and emission optical signals. Additionally, the epoxy acted as a thermal link between the $\mathrm{O}_{2}$ sensing film and the electronics (LEDs, photodiode and temperature sensor), which, due to the small size and mass of the sensor head, allows fast thermal equilibration, resulting in precise local temperature readings of the skin surface, film, and LEDs with a short response time.

\subsubsection{Control electronics}

The PCBs were designed with the KiCad software and fabricated with standard fiberglass substrates (OSH Park, Portland, Oregon, USA). A flexible ribbon cable (Molex, Lisle, IL, USA) connects the sensor head to the control electronics, consisting of a custom designed PCB with electronics built around a Particle Photon or Argon microcontroller board (Particle, San Francisco, CA, USA) with Bluetooth Low Energy (BLE) and/or WiFi connectivity, powered by a rechargeable lithium polymer battery (see 1 ). The microcontroller communicates via I2C with a 12 bit analog-to-digital converter (ADC) (Texas Instruments, Dallas, TX, USA), which samples the reference signal, the photodiode signal and the thermistor voltage through three differential voltage channels. With the $5 \mathrm{~V}$ reference provided, the $\mathrm{ADC}$ achieves a resolution of $1.2 \mathrm{mV} / \mathrm{bit}$. The LEDs are modulated by a sine-like wave voltage signal (the reference signal) of programmable frequency $\left(f_{r}\right)$. A reference frequency of $f_{r}=796 \mathrm{~Hz}$ was used for this study. The reference signal is obtained by low pass (LP) filtering (4-pole with corner or roll-off freq. $f_{c}=f_{r}$ ) and amplifying (gain $x 2$ ) a square wave (PWM) output supplied by the micro-controller (see Fig. S4(a)(b)). The filter and gain were chosen to achieve an optimal depth-modulation of the LEDs, which produce a high contrast, sine-like emission with a very low minimum output and a high brightness maximum. The LP filter approach was chosen as a simple way to obtain a sine-like reference signal using only passive components. Using a function generator the LEDs were found to produce brighter emission for frequencies below $1 \mathrm{kHz}$. Additionally, as shown in Fig. S2(c), the LEDs also exhibit a phosphorescence hump in the spectral region in which the oxygen 
sensing dye emits, and the ratio of blue emission to phosphorescence was highest for slower frequencies. The $796 \mathrm{~Hz}$ value is arrived at by using $\mathrm{R}=5 \mathrm{k} \Omega, \mathrm{C}=0.1 \mu F$ so $f_{c}=1 /(2 \pi R C)$.

The photodiode signal is amplified by a transimpedance amplifying circuit following the application note in Ref. [50]. The TIA gain was chosen to maximize the use of the dynamic range of the ADC (i.e. the ADC counts are high but do not saturate at maximum phosphorescence signal) once the sensor head geometry, porphyrin concentration and reference signal frequency were fixed. With this resistor fixed at $2.5 \mathrm{M} \Omega$, and a feedback capacitor of $C=0.1 p F$, we achieve a bandwidth of $636 \mathrm{kHz}$. More information on the DC and AC characterization of the TIA, as well as ADC inputs and outputs can be found in Fig. S4-6.

Temperature was measured by a two-resistor circuit composed of a thermistor of $R_{1}=10 \mathrm{k} \Omega$ and B-value of 3650 (see Fig. S10) and a reference resistor $\left(R_{2}=5 k \Omega\right.$ ), and using the Steinhart-Hart thermistor equation [51]. A 3D printed casing (Fig. 1) was designed to house the electronics and allow the secure fastening of the device onto a limb through an elastic band or strap.

\subsubsection{Calibration}

The calibrations were carried out in a sealed calibration chamber in which the temperature and $\mathrm{pO}_{2}$ can be controlled by a hot plate and by varying the mix of nitrogen and air gas being fed into a gas mixer, as shown in Fig. S3. The partial oxygen pressure in the chamber was measured with a commercial oxygen sensor (PreSens, Regensburg, Germany).

\subsubsection{Data collection}

To obtain a single measurement of $\mathrm{pO}_{2}$ the modulated LEDs are flashed for around 0.2 seconds, during which 1000 measurements of \{time, voltage pairs are recorded for both reference and photodiode signals, where the time is measured in microseconds. The LED flash duration is determined by the ADC sampling rate and the desired number of acquired data points. The number of data points (1000) was chosen as it provides the amount of detail required to extract the phase and intensity values with precision. By using the modulo operator, we use the time variable $t^{\prime}=\bmod (t, T)$ with $T=1 / f_{r}$, which allows us to reconstruct a single oscillation of the reference and photodiode signal with an effectively high time-resolution (see Fig. S6 and 7). Data was collected through a Python [52] script on a PC through USB serial port or through Bluetooth.

\subsection{Data analysis}

\subsubsection{Extracting phase and intensity}

The reference signal is dominated by a sine at the fundamental frequency, but higher order $o d d$ harmonics $(3 f, 5 f, 7 f, \ldots)$ contribute to the signal, which are not fully filtered out from the square-wave PWM source. Plotting the Fast Fourier transform (FFT) of the reference signal (see Fig. S7(c)) shows that the amplitude of harmonics $3 f, 5 f, 7 f$ is only $2.3 \%, 0.8 \%$ and $0.3 \%$ that of the fundamental frequency. We found that including terms up to $7 f$ improves the accuracy of the fit and allows determining the phase with high precision and reproducibility. As can be seen (see Fig. S7(c)), the photodiode signal requires including even harmonics $(2 f, 4 f, \ldots)$ as well, as they are present in the phosphorescence due to the non-linear nature of LEDs [53].

The following function is fit to the reference and photodiode signals using Multiple Linear Regression in the matrix form [44]:

$$
y(t)=\beta_{0}+\sum_{i}\left(\beta_{2 i-1} \cos (i 2 \pi f t)+\beta_{2 i} \sin (i 2 \pi f t)\right), i \in[1,2,3,4,5,7, \ldots]
$$




\section{Biomedical Optics EXPRESS}

where $\cos (2 \pi f t+\theta)=\cos \theta \cos (2 \pi f t)-\sin \theta \sin (2 \pi f t)$. The least squares coefficients $\beta_{i}$ allow for the calculation of the intensity $(I)$ and phase $(\theta)$ of the fundamental frequency:

$$
\begin{gathered}
I=\sqrt{\beta_{1}^{2}+\beta_{2}^{2}} \\
\theta=\arccos \left(\beta_{1} / I\right)
\end{gathered}
$$

The result of the fitting can be seen in Fig. S8. The data analysis was carried out using GNU Octave [54] and re-written to $\mathrm{C}++$ for the device to perform the calculations on-board.

\subsubsection{Error calculation}

The standard error of the least squares coefficients is obtained from the variance-covariance matrix of the fitting parameters. The standard error of $\theta$ and $I$ is calculated by error propagation. The $95 \%$ confidence interval $\left(95 \%\right.$ C.I) of the $\mathrm{pO}_{2}$ values obtained from both phase and intensity measurements was calculated by estimating the standard error of $\mathrm{pO}_{2}$ using the Stern-Volmer equation and error propagation.

\section{Funding}

Henry M. Jackson Foundation (HU0001-17-2-009); Air Force Office of Scientific Research (FA9550-17-1-0277).

\section{Acknowledgements}

We thank J.Q. Nguyen for constructive criticism of the manuscript and the members of the Evans laboratory for their help throughout this research project. This work was supported by the Military Medical Photonics Program, FA9550-17-1-0277 and the Military Medicine Transforming Technology Collaborative, HU0001-17-2-009.

\section{Disclosures}

The authors declare no conflicts of interest.

See Supplement 1 for supporting content.

\section{References}

1. M. S. Butler and P. E. Luebbers, "Health and fitness wearables," in Wearable Technologies: Concepts, Methodologies, Tools, and Applications, (IGI Global, 2018), pp. 30-50.

2. D. R. Seshadri, R. T. Li, J. E. Voos, J. R. Rowbottom, C. M. Alfes, C. A. Zorman, and C. K. Drummond, "Wearable sensors for monitoring the physiological and biochemical profile of the athlete," npj Digital Medicine 2(1), 72 (2019).

3. Z. Li, N. Navarro-Alvarez, E. J. Keeley, N. H. Nowell, B. M. Goncalves, C. A. Huang, and C. L. Evans, "Non-invasive monitoring of skin inflammation using an oxygen-sensing paint-on bandage," Biomed. Opt. Express 8(10), 4640-4651 (2017).

4. J. Q. Nguyen, H. L. Marks, T. Everett, T. Haire, A. Carlsson, R. Chan, and C. L. Evans, "Early visualization of skin burn severity using a topically applied dye-loaded liquid bandage," Sci. Rep. 10(1), 9314 (2020).

5. T. E. McMillan, W. T. Gardner, A. H. Schmidt, and A. J. Johnstone, "Diagnosing acute compartment syndrome-where have we got to?" Int. Orthop. 43(11), 2429-2435 (2019).

6. L. R. McPhail, L. T. Cooper, D. O. Hodge, M. E. Cabanel, and T. W. Rooke, "Transcutaneous partial pressure of oxygen after surgical wounds," Vasc. Med. 9(2), 125-127 (2004).

7. Y. Khan, D. Han, A. Pierre, J. Ting, X. Wang, C. M. Lochner, G. Bovo, N. Yaacobi-Gross, C. Newsome, and R. Wilson, “A flexible organic reflectance oximeter array," Proc. Natl. Acad. Sci. 115(47), E11015 (2018).

8. M. Vegfors, L. G. Lindberg, and C. Lennmarken, "The influence of changes in blood flow on the accuracy of pulse oximetry in humans," Acta Anaesthesiol. Scand. 36(4), 346-349 (1992).

9. N. R. Clay and C. M. Dent, "Limitations of pulse oximetry to assess limb vascularity," J. Bone Jt. Surg., Br. Vol 73-B(2), 344 (1991). 
10. H. H. Moosa, M. S. Makaroun, A. B. Peitzman, D. L. Steed, and M. W. Webster, "Tcpo2 values in limb ischemia: Effects of blood flow and arterial oxygen tension,” J. Surg. Res. 40(5), 482-487 (1986).

11. P. Kranke, M. H. Bennett, M. Martyn-St James, A. Schnabel, S. E. Debus, and S. Weibel, "Hyperbaric oxygen therapy for chronic wounds," Cochrane Database of Systematic Reviews (2015).

12. W. L. Rumsey, J. M. Vanderkooi, and D. F. Wilson, "Imaging of phosphorescence: a novel method for measuring oxygen distribution in perfused tissue," Science 241(4873), 1649-1651 (1988).

13. J. M. Vanderkooi, G. Maniara, T. J. Green, and D. F. Wilson, "An optical method for measurement of dioxygen concentration based upon quenching of phosphorescence," J. Biol. Chem. 262(12), 5476-5482 (1987).

14. P. Babilas, P. Lamby, L. Prantl, S. Schreml, E. M. Jung, G. Liebsch, O. S. Wolfbeis, M. Landthaler, R.-M. Szeimies, and C. Abels, "Transcutaneous po2 imaging during tourniquet-induced forearm ischemia using planar optical oxygen sensors," Ski. Res. Technol. 14(3), 304-311 (2008).

15. P. Babilas, G. Liebsch, V. Schacht, I. Klimant, O. S. Wolfbeis, R.-M. Szeimies, and C. Abels, "In vivo phosphorescence imaging of p o 2 using planar oxygen sensors," Microcirculation 12(6), 477-487 (2005).

16. E. Bailey Jr and G. Rollefson, "The determination of the fluorescence lifetimes of dissolved substances by a phase shift method," J. Chem. Phys 21(8), 1315-1322 (1953).

17. E. R. Menzel and Z. D. Popovic, "Picosecond-resolution fluorescence lifetime measuring system with a cw laser and a radio," Rev. Sci. Instrum. 49(1), 39-44 (1978).

18. R. J. Meier, S. Schreml, X.-d. Wang, M. Landthaler, P. Babilas, and O. S. Wolfbeis, "Simultaneous photographing of oxygen and ph in vivo using sensor films," Angewandte Chemie Int. Ed. 50(46), 10893-10896 (2011).

19. S. Schreml, R. J. Meier, O. S. Wolfbeis, T. Maisch, R.-M. Szeimies, M. Landthaler, J. Regensburger, F. Santarelli, I. Klimant, and P. Babilas, "2d luminescence imaging of physiological wound oxygenation," Exp. Dermatol. 20(7), 550-554 (2011)

20. Z. Li, E. Roussakis, P. G. Koolen, A. M. Ibrahim, K. Kim, L. F. Rose, J. Wu, A. J. Nichols, Y. Baek, R. Birngruber, G. Apiou-Sbirlea, R. Matyal, T. Huang, R. Chan, S. J. Lin, and C. L. Evans, "Non-invasive transdermal two-dimensional mapping of cutaneous oxygenation with a rapid-drying liquid bandage," Biomed. Opt. Express 5(11), 3748-3764 (2014).

21. S. Schreml, R. Meier, M. Kirschbaum, S. Kong, S. Gehmert, O. Felthaus, S. Küchler, J. Sharpe, K. Wöltje, and K. Weiß, et al., "Luminescent dual sensors reveal extracellular ph-gradients and hypoxia on chronic wounds that disrupt epidermal repair." (2014).

22. P. G. Koolen, Z. Li, E. Roussakis, M. A. Paul, A. Ibrahim, R. Matyal, T. Huang, C. L. Evans, and S. J. Lin, "Oxygen-sensing paint-on bandage: calibration of a novel approach in tissue perfusion assessment," Plast. Reconstr. Surg. 140(1), 89-96 (2017).

23. I. Costanzo, D. Sen, and U. Guler, "A prototype towards a transcutaneous oxygen sensing wearable," in 2019 IEEE Biomedical Circuits and Systems Conference (BioCAS), (2019), pp. 1-4.

24. S. B. Bambot, R. Holavanahali, J. R. Lakowicz, G. M. Carter, and G. Rao, "Phase fluorometric sterilizable optical oxygen sensor," Biotechnol. Bioeng. 43(11), 1139-1145 (1994).

25. G. A. Holst, T. Köster, E. Voges, and D. W. Lübbers, "FLOX-an oxygen-flux-measuring system using a phasemodulation method to evaluate the oxygen-dependent fluorescence lifetime," Sens. Actuators, B 29(1-3), 231-239 (1995).

26. M. Stücker, P. Altmeyer, A. Struk, K. Hoffmann, L. Schulze, A. Röchling, and D. W. Lübbers, "The transepidermal oxygen flux from the environment is in balance with the capillary oxygen supply," J. Invest. Dermatol. 114(3), 533-540 (2000).

27. M. Stücker, A. Struk, P. Altmeyer, M. Herde, H. Baumgärtl, and D. W. Lübbers, "The cutaneous uptake of atmospheric oxygen contributes significantly to the oxygen supply of human dermis and epidermis," J. Physiol. 538(3), 985-994 (2002).

28. C. J. Lim, S. Lee, J. H. Kim, H. J. Kil, Y. C. Kim, and J. W. Park, "Wearable, Luminescent Oxygen Sensor for Transcutaneous Oxygen Monitoring," ACS Appl. Mater. Interfaces 10(48), 41026-41034 (2018).

29. W. van Weteringen, T. G. Goos, T. van Essen, C. Ellenberger, J. Hayoz, R. C. de Jonge, I. K. Reiss, and P. M. Schumacher, "Novel transcutaneous sensor combining optical tcPO2 and electrochemical tcPCO2 monitoring with reflectance pulse oximetry," Med. Biol. Eng. Comput. 58, 239-247 (2019).

30. Y. Katayama, Y. Fujioka, and K. Tsukada, "Development of a patch-type flexible oxygen partial pressure sensor," IEEE J. Transl. Eng. Health Med. 8, 1-7 (2020)

31. M. F. Montero-Baker, K. Y. Au-Yeung, N. A. Wisniewski, S. Gamsey, L. Morelli-Alvarez, J. L. Mills, M. Campos, and K. L. Helton, "The First-in-Man "si Se Puede" Study for the use of micro-oxygen sensors (MOXYs) to determine dynamic relative oxygen indices in the feet of patients with limb-threatening ischemia during endovascular therapy," J. Vasc. Surg. 61(6), 1501-1510.e1 (2015).

32. N. A. Wisniewski, S. P. Nichols, S. J. Gamsey, S. Pullins, K. Y. Au-Yeung, B. Klitzman, and K. L. Helton, "Tissue-integrating oxygen sensors: continuous tracking of tissue hypoxia," in Oxygen Transport to Tissue XXXIX, (Springer, 2017), pp. 377-383.

33. G. J. Kintz, W. A. McMillan, and N. A. Wisniewski, "Apparatus and methods for detecting optical signals from implanted sensors," (2019). US Patent 10,219,729.

34. Z. Yang, "Implantable biosensor," (2005). US Patent App. 10/631,908. 
35. E. Roussakis, Z. Li, N. H. Nowell, A. J. Nichols, and C. L. Evans, "Bright,"clickable" porphyrins for the visualization of oxygenation under ambient light," Angew. Chem. Int. Ed. 54(49), 14728-14731 (2015).

36. R. Chen, F. Formenti, H. McPeak, A. N. Obeid, C. Hahn, and A. Farmery, "Experimental investigation of the effect of polymer matrices on polymer fibre optic oxygen sensors and their time response characteristics using a vacuum testing chamber and a liquid flow apparatus," Sens. Actuators, B 222, 531-535 (2016).

37. E. Roussakis, J. P. Cascales, H. L. Marks, X. Li, M. Grinstaff, and C. L. Evans, "Humidity-insensitive tissue oxygen tension sensing for wearable devices," Photochem. Photobiol. 96(2), 373-379 (2020).

38. X.-d. Wang and O. S. Wolfbeis, "Optical methods for sensing and imaging oxygen: materials, spectroscopies and applications," Chem. Soc. Rev. 43(10), 3666-3761 (2014).

39. H.-P. Haar and M. Hauser, "Phase fluorometer for measurement of picosecond processes," Rev. Sci. Instrum. 49(5), 632-633 (1978).

40. E. Gratton and R. Lopez-Delgado, "Measuring fluorescence decay times by phase-shift and modulation techniques using the high harmonic content of pulsed light sources," Nuov. Cim. B 56(1), 110-124 (1980).

41. E. Gratton and M. Limkeman, "A continuously variable frequency cross-correlation phase fluorometer with picosecond resolution,” Biophys. J. 44(3), 315-324 (1983).

42. K. W. Berndt and J. R. Lakowicz, "Electroluminescent lamp-based phase fluorometer and oxygen sensor," Anal. Biochem. 201(2), 319-325 (1992).

43. B. H. Weigl, A. Holobar, W. Trettnak, I. Klimant, H. Kraus, P. O’Leary, and O. S. Wolfbeis, "Optical triple sensor for measuring ph, oxygen and carbon dioxide," J. Biotechnol. 32(2), 127-138 (1994).

44. M. S. Pedersen, B. Baxter, B. Templeton, C. Rishøj, D. L. Theobald, E. Hoegh-rasmussen, G. Casteel, J. B. Gao, K. Dedecius, K. Strim, L. Christiansen, L. K. Hansen, L. Wilkinson, L. He, M. Bar, O. Winther, P. Sakov, S. Hattinger, K. B. Petersen, and C. Rishø j, "The matrix cookbook," Matrix (2008).

45. O. Stern and M. Volmer, “Über die abklingzeit der fluoreszenz," Phys. Z 20, 183-188 (1919).

46. D. P. F. Friedl, "Investigating the Transfer of Oxygen at the Wavy Air-Water Interface under Wind-Induced Turbulence." Thesis (2013).

47. L. Rosso and V. C. Fernicola, "Time- and frequency-domain analyses of fluorescence lifetime for temperature sensing," Rev. Sci. Instrum. 77(3), 034901 (2006).

48. A. D. Elder, J. H. Frank, J. Swartling, X. Dai, and C. F. Kaminski, "Calibration of a wide-field frequency-domain fluorescence lifetime microscopy system using light emitting diodes as light sources," J. Microsc. 224(2), 166-180 (2006).

49. B. Leenstra, J. Wijnand, B. Verhoeven, O. Koning, M. Teraa, M. C. Verhaar, and G. J. de Borst, "Applicability of transcutaneous oxygen tension measurement in the assessment of chronic limb-threatening ischemia," Angiology 71(3), 208-216 (2020).

50. J. Caldwell, "1 mhz single-supply photodiode amplifier reference design,” TI Application Note TIDU535 pp. 1-19 (2014).

51. J. S. Steinhart and S. R. Hart, "Calibration curves for thermistors," Deep-Sea Research and Oceanographic Abstracts (1968).

52. G. Van Rossum and F. L. Drake, The Python Language Reference Manual (Network Theory Ltd., 2011).

53. Ł. Putz, K. Bednarek, and R. Nawrowski, "Disturbances generated by lighting systems with led lamps and the reduction in their impacts," Appl. Sci. 9(22), 4894 (2019).

54. S. H. John, W. Eaton, David Bateman, and R. Wehbring, GNU Octave Version 3.8.1 Manual: A High-level Interactive Language for Numerical Computations (CreateSpace Independent Publishing Platform, 2014). ISBN 1441413006. 